\title{
Duodenal Hemorrhage, CTCAE
}

National Cancer Institute

\section{Source}

National Cancer Institute. Duodenal Hemorrhage, CT CAE. NCI Thesaurus. Code

C143414.

A disorder characterized by bleeding from the duodenum. 